Article

\title{
Damping Estimation of an Eight-Story Steel Building Equipped with Oil Dampers
}

\author{
Pengchao Yang ${ }^{1}$, Songtao Xue ${ }^{1,2}$, Liyu Xie ${ }^{1, * \mathbb{D}}$ and Miao Cao ${ }^{2}$ \\ 1 Department of Disaster Mitigation for Structures, Tongji University, Shanghai 200092, China; \\ murphy_ypc@126.com (P.Y.); xue@tongji.edu.cn (S.X.) \\ 2 Department of Architecture, Tohoku Institute of Technology, Sendai 982-8577, Japan; caomiao@tohtech.ac.jp \\ * Correspondence: liyuxie@tongji.edu.cn; Tel.: +86-21-6598-2390
}

Received: 26 November 2020; Accepted: 13 December 2020; Published: 16 December 2020

\begin{abstract}
The damping estimation of an eight-story steel building equipped with oil dampers is examined, carried out by adopting a proposed framework, which consists of an enhanced strain-energy method and an improved direct method for model updating. The building is located at Tohoku Institute of Technology and is equipped with a structural monitoring system that measures its seismic response, including floor acceleration and displacement and force of oil dampers. The enhanced strain-energy method is first developed and employed to assess the supplemental damping and stiffness provided by oil dampers, herein quantified in the form of equivalent damping ratios and natural frequencies. Then, modal characteristics extracted from the earthquake measurements are modified accordingly and utilized for the building model updating, in which mass and stiffness matrices are corrected by the improved direct method. The updated model accurately reproduces the target modal data, especially measured mode participation factors, and is further used for the building response predictions. Through prediction validations, the precision of the modified modal parameters is verified. Finally, a large earthquake event is chosen to demonstrate the effectiveness of the proposed framework for the damping estimation of the investigated building.
\end{abstract}

Keywords: equivalent damping ratios; strain-energy method; direct updating method; oil dampers; earthquake measurements

\section{Introduction}

In the past few decades, many damping devices have been developed and applied in civil engineering structures, especially in earthquake-prone regions [1-3]. Viscous dampers generally represent a broad class of passive energy dissipation devices and have emerged as one of the most popular ones. By providing additional damping and energy dissipation, they can efficiently suppress earthquake-induced vibrations and, therefore, limit the damage to structural and non-structural components [4-6]. To better understand the damping properties of such dampers, many experimental studies have been conducted, including damper element tests $[7,8]$ and shaking table tests of small-scale and full-scale structures with viscous dampers [4,5]. However, studies focusing on in-service structures with such dampers when subjected to large earthquakes are relatively rare $[9,10]$. This practical issue requires accurate state quantification for dampers and structure itself, which holds a key position in structural health, safety, and risk assessments [11,12]. This paper presents a study on the damping estimation of an eight-story building structure equipped with oil dampers through a proposed framework, which consists of an enhanced strain-energy method and an improved direct method for model updating.

The strain-energy method is usually adopted at the design stage of passively controlled structures to assess the damping effect of added dampers quantitatively. Specifically, it incorporates the 
supplemental damping provided by dampers into modal damping ratios, which are frequently referred to as equivalent damping ratios and are defined as the ratios of energy dissipated by dampers and stored in structure system. Zhang and Soong [13] provided a procedure to quantify the damping effect of viscoelastic dampers and showed their effectiveness in attenuating structural seismic response. Chang et al. [14] carried out a comprehensive experimental program on a 2/5-scale five-story steel frame with viscoelastic dampers and applied this procedure to assess the equivalent damping ratios for measured modes. Ji et al. [15] conducted a series of shaking table tests on a full-scale five-story steel building with three types of passive dampers, including viscous dampers and oil dampers. Using the strain-energy method and system identification, they investigated the damping characteristics of those dampers and presented simplified estimations for their supplemental damping ratios.

In this study, the strain-energy method is adopted to estimate the supplemental damping ratios provided by oil dampers for the investigated building under earthquake excitations. Additionally, it is notable that, except for the supplement damping, dampers can contribute to the increased lateral stiffness of the entire structure [14-17]. This stiffness contribution, usually not large, may affect structural dynamic behavior, such as increasing natural frequencies. For evaluating such modal changes, an enhanced strain-energy method is proposed herein, quantifying the damper stiffness in the form of equivalent natural frequencies, i.e., the natural frequency changes of structures with and without dampers. Besides, note that the strain-energy method adopts assumptions of harmonic motion and mode-proportional deformation for dampers and structure itself, which violate their realistic situation during an earthquake event [15]. Consequently, the estimated equivalent damping ratios and natural frequencies may be questionable and, therefore, need to be verified under earthquake situations. In this study, it is achieved through model updating and prediction validations.

Model updating using dynamic measurements has been a topic of extensive focus for many engineering principles, including civil engineering [18]. In structural dynamics community, it is well-understood that the predictions from an initial structural model are frequently inconsistent with those observed from its real-world counterpart [19]. These predictions could be modal properties, time histories, or frequency response functions. The objective of model updating is to reduce such discrepancy, improving the correlation between numerical models and their real-world counterparts. Generally, model updating belongs to the family of inverse problems, and, in most practical applications, it can be approached from an optimization perspective. In recent decades, a significant number of model updating methods have been developed. Literature review for this topic is not presented here since is beyond the scope of this study. The interested reader is referred to references [19-21]. This paper is concerned only with the so-called direct updating methods because of their particular features, the resulting updated model reproduces exactly the target/measured modal data, including natural frequencies and mode shapes. By taking advantage of such features, the damper stiffness effect on structural dynamic behavior can be easily captured in model updating.

The direct updating methods are essentially analytical methods which do not require iterations. In those methods, modal orthogonality, symmetry, and eigenvalue equation are usually imposed in correcting mass matrix or stiffness matrix or both, and minimum possible changes in updated matrices are achieved by using Lagrange multipliers or generalized inverse. Baruch and Bar-Itzack [22] first formulated a procedure using Lagrange multipliers to correct stiffness matrix to satisfy modal orthogonality and the eigenvalue equation with nominal mass matrix. Berman [23] questioned the assumption that nominal mass matrix is correct and developed a new procedure to modify mass matrix under modal orthogonality constraint. Later, Berman and Nagy [24] integrated these two procedures into the so-called analytical model improvement technique to update a long-exposure facility structure. Based on this research, Caesar and Peter [25] further described two direct methods for model updating using modal data. Wei [26] considered the interaction between mass and stiffness matrices in their updating processes and proposed a united procedure to modify them simultaneously using the vibration test data. Yue [27] developed an algorithm turning model updating to a forward problem, in which a transformation matrix is only required to update mass and stiffness matrices. Lee and Eun [28] 
employed the Moore-Penrose inverse, rather than Lagrange multipliers, to reformulate the model updating problem. But the resulting corrected stiffness matrices lose symmetricity. Yang and Chen [29] established a simple procedure to maintain the physical meanings in mass and stiffness matrices corrections, which allows the updated model to be compatible with the measured natural frequencies. All of these methods discussed above are computationally efficient and can be easily applied to large structural models $[24,27,29]$. However, for applications in earthquake engineering, a problem arises that the predicted mode participation factors by updated models may not be consistent with those identified from earthquake measurements. To address this problem, an improved Berman-Nagy method is developed, using a new procedure to correct nominal mass matrix under the constraints of both modal orthogonality and mode participation factors.

A large earthquake event is chosen to demonstrate the effectiveness of the proposed framework for the damping estimation of the investigated building. The enhanced strain-energy method is first employed to calculate the equivalent damping ratios and natural frequencies provided by oil dampers, which are modeled by a linear Maxwell model consisting of a spring element connected in series with a dashpot element $[7,15]$. Then, the modal characteristics extracted from earthquake measurements are modified accordingly. The modified parameters are ultimately the data utilized to update an initial finite element model of the building by using the improved Berman-Nagy method. Finally, through prediction validations, the precision of the modified parameters as well as the estimated equivalent damping ratios and natural frequencies are verified. The remaining of this paper is organized as follows: in Section 2, the investigated building and its structural monitoring system are introduced. Section 3 presents the details of the enhanced strain-energy method and the improved Berman-Nagy method. The results of damping estimation for the building during the selected earthquake are illustrated in Section 4. Section 5 provides the conclusions of this study.

\section{Building Description and Its Structural Monitoring System}

\subsection{Overview of the Building}

The investigated structure, as shown in Figure 1, is the administration building of Tohoku Institute of Technology, located in Sendai, Japan. It was constructed in 2003 and is steel framed with precast concrete slabs. The building has a rectangular plan of dimensions 48 by $9.6 \mathrm{~m}$, while the total height is $34.2 \mathrm{~m}$, with eight stories above the ground. The story height is $3.8 \mathrm{~m}$, except for the first floor, which has a height of $8 \mathrm{~m}$ since the first two stories merge for a large public place.

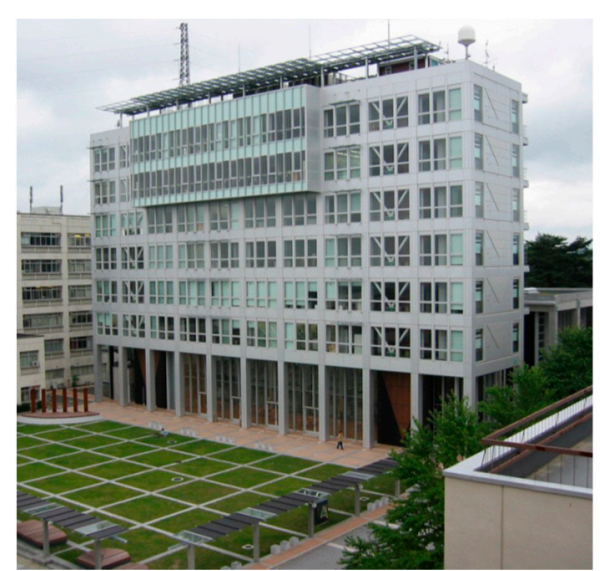

(a)

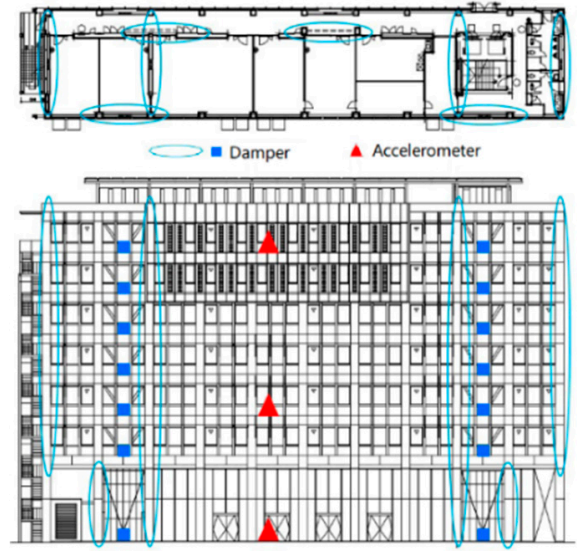

(b)

Figure 1. The administration building: (a) front view; (b) allocation of oil dampers and accelerometers.

The building was designed according to the Japanese Building Standard Law without considering the incorporation of damping devices. For enhanced seismic protection, a total of 56 oil dampers were additionally installed in the building. Each floor has 8 oil dampers equally distributed in the 
two orthogonal directions, as shown in Figure 1b. Two types of such oil dampers are fixed on floors and are connected with the adjacent upper floors through V-type steel braces. These dampers have different orifice specifications and stroke limits for the 1st floor (Type I) and 3rd to 8th floor (Type II), as described in Figure 2 using notation $1 \mathrm{~F}$ and 3-8F.

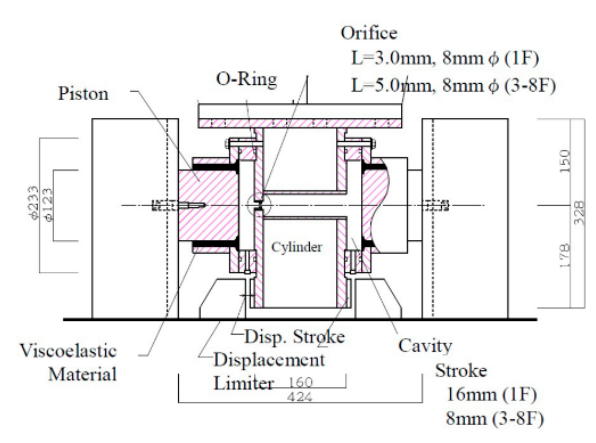

Figure 2. Damper configuration details.

The oil damper, designed by Kawamata et al. [30], consists of a central cylinder and a pair of pistons. The gaps between them are sealed by using viscoelastic polymers. The pistons are fixed on a U-type abutment on floors, and the central cylinder is attached to a V-type brace moving between the two pistons. Figure 2 shows the detailed layout and dimensions of the installed oil dampers. The relative movements between the cylinder and the pistons create the damper resisting force, which consists of two parts: the pressure resistance coming from the flowing infilled oil through a small orifice and the viscoelastic resisting force caused by the shearing deformations of the sealing materials.

Forced vibration tests were conducted after completing the building frame in June 2003, to investigate the dynamic characteristics of the structure with and without oil dampers. The identification results from vibration tests showed an apparent increase in natural frequencies, the fundamental frequencies increased from 1.05 to $1.12 \mathrm{~Hz}$ along the transversal direction and 1.02 to $1.10 \mathrm{~Hz}$ along the longitudinal direction. The increased natural frequencies stem from the additional stiffness provided by oil dampers, which motives this study to investigate such stiffness effect during earthquakes.

\subsection{Structural Monitoring System}

A structural monitoring system is installed in the building to monitor its seismic motions. The monitoring system consists of three two-directional acceleration sensors for measuring floor acceleration and four pairs of load cell and displacement transducer for recording the force and displacement responses of oil dampers. These accelerometers are placed near the central locations of the 1st, 4th, and 8th floor plans, as shown in Figure 1b. They are utilized to measure the floor translational motions along with the two horizontal directions. Four oil dampers, as representatives of the two-type dampers, were selected to be monitored. Specifically, dampers installed on the 1st and 8th floors were chosen, with each floor having two monitored oil dampers along with its two principal directions.

Figure 3 shows a typical set of measured seismic responses, including floor acceleration and force and displacement of oil dampers in the building translational direction. This data set was recorded during a large earthquake with a moment magnitude of 7.0, which happened on 26 May 2003. The force-displacement hysteretic curve of the oil dampers shows their damping and stiffness properties during this earthquake. The earthquake measurements obtained from this earthquake are utilized in this study to demonstrate the damping estimation process for the building. 


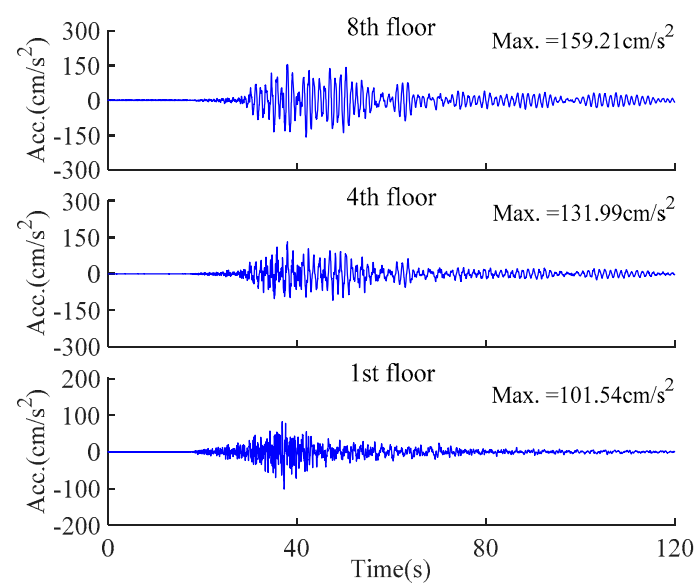

(a)
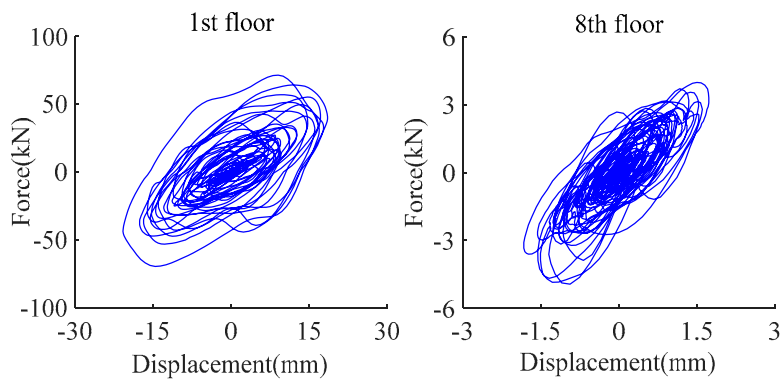

(b)

Figure 3. A typical set of earthquake measurements of the building: (a) Accelerations of monitored floors; (b) Force and displacement of monitored oil dampers.

\section{A Framework for Damping Estimation of Passively Controlled Structures}

This section proposes a general framework for the damping estimation of passively controlled structures. It consists of two newly developed methods, namely, an enhanced strain-energy method and an improved Berman-Nagy method. The former method aims to quantify the additional damping and stiffness provided by dampers in the forms of equivalent damping ratios and natural frequencies. In particular, simple estimation formulas are derived for the case added dampers modeled by linear Maxwell models. The latter method is devoted to correcting the nominal mass and stiffness matrices of passively controlled structures themself using measured modal data, including natural frequencies, mode shapes, as well as mode participation factors.

\subsection{An Enhanced Strain-Energy Method}

\subsubsection{Estimation of Equivalent Damping Ratios}

The traditional strain-energy method assumes passively controlled structure vibrates in harmonic motion with a frequency consistent with one of their natural frequencies and deflects proportionally to the corresponding mode shape. Based on these assumptions, the equivalent damping ratio provided by added dampers can be estimated by

$$
\xi_{\text {eq, }, i}=\frac{1}{4 \pi} \frac{E_{D, i}}{E_{S, i}}
$$

where $\xi_{\text {eq }, i}$ is the equivalent damping ratio for the $i$ th mode, $E_{D, i}$ is the energy dissipated by dampers in one cycle of motion, $E_{S, i}$ is the corresponding maximum strain energy stored in the entire structure system, which is estimated by

$$
E_{S, i}=\frac{1}{2} \Delta_{i}^{2} \boldsymbol{\phi}_{i}^{T} \mathbf{K} \boldsymbol{\phi}_{i}=\frac{1}{2} \Delta_{i}^{2} \omega_{i}^{2}
$$


where $\mathbf{K}$ is the stiffness matrix of the entire structure, $\omega_{i}$ and $\phi_{i}$ are, respectively, the $i$ th circular frequency and mass-normalized mode shape, $\Delta_{i} \boldsymbol{\phi}_{i}$ denotes the maximum structural deformation vector in one cycle of motion, $\Delta_{i}$ is an unknown factor determining the $i$ th mode vibration amplitude.

Formulation of $E_{D, i}$ depends on damper types. For the case added dampers modeled by linear Maxwell models, $E_{D, i}$ is given by [15]

$$
E_{D, i}=\pi \sum_{j} \frac{\omega_{i}}{1+\tau_{d, j}^{2} \omega_{i}^{2}} c_{d, j} \Delta_{i}^{2} \phi_{i, d j}^{2}
$$

where $\tau_{d, j}=c_{d, j} / k_{d, j}$ is the relax time for the $j$ th damper, $c_{d, j}$ and $k_{d, j}$ are the damping and stiffness coefficients, respectively. $\phi_{i, d j}$ is the axial deformation of the $j$ th damper in the $i$ th mass-normalized mode shape.

Substitute Equations (2) and (3) into Equation (1) yields the $i$ th equivalent damping ratio. For simplifying this formula, a coordinate transformation, $\Delta_{i} \boldsymbol{\phi}_{i, d}=\mathbf{T}_{D} \Delta_{i} \boldsymbol{\phi}_{i}$, is introduced between damper deformation $\Delta_{i} \boldsymbol{\phi}_{i, d}$ (local damper coordinate system) and structure deformation $\Delta_{i} \boldsymbol{\phi}_{i}$ (global coordinate system). $\mathbf{T}_{D}$ is a predefined transformation matrix determined by damper configure details. By substituting such transformation into Equation (3), we can get

$$
E_{D, i}=\pi \omega_{i} \Delta_{i}^{2} \boldsymbol{\phi}_{i}^{\mathrm{T}} \widetilde{\mathbf{C}}_{D, i} \boldsymbol{\phi}_{i}
$$

where the matrix $\widetilde{\mathbf{C}}_{D, i}$ is given by

$$
\widetilde{\mathbf{C}}_{D, i}=\mathbf{T}_{D}^{\mathrm{T}} \operatorname{diag}\left\{\frac{c_{d, j}}{1+\tau_{d, j}^{2} \omega_{i}^{2}}\right\} \mathbf{T}_{D}
$$

It can be regarded as the equivalent damping matrix for the $i$ th mode provided by added dampers. The equivalent damping ratio is ultimately expressed by

$$
\xi_{e q, i}=\frac{\boldsymbol{\phi}_{i}^{T} \widetilde{\mathbf{C}}_{D, i} \boldsymbol{\phi}_{i}}{2 \omega_{i}}
$$

Notably, such an expression follows the classical definition of modal damping ratios and assign strong physically meanings to the calculations of equivalent damping ratios.

\subsubsection{Estimation of Equivalent Natural Frequencies}

An energy approach is presented here to quantify damper stiffness by equivalent natural frequencies. It assumes that the added dampers of a structure do not affect its mode shapes, which can be justified by the fact that damper stiffness is usually minor compared with structure stiffness $[15,31]$. The basic idea of this approach is based on Equation (2), which establishes a relationship between strain energy and natural frequency, and the critical point in this approach is to determine the distribution of strain energy stored in the entire structure. Specifically, for the $i$ th mode, the strain energy stored in structure itself may be expressed by

$$
E_{S, i}^{S}=\left(1-\frac{E_{D, i}^{k}}{E_{S, i}}\right) E_{S, i}=\left(1-\eta_{D, i}\right) E_{S, i}
$$

where $\eta_{D, \text { and }}$ is a strain-energy ratio, and $E_{D, i}^{k}$ is the maximum strain energy stored in dampers in one cycle of motion. It has a closed-form solution for oil dampers modeled by linear Maxwell models as

$$
E_{D, i}^{k}=\frac{1}{2} \sum_{j} \frac{\tau_{d, j}^{2} \omega_{i}^{2}}{1+\tau_{d, j}^{2} \omega_{i}^{2}} k_{d, j} \Delta_{i}^{2} \phi_{i, d j}^{2}=\frac{1}{2} \Delta_{i}^{2} \boldsymbol{\phi}_{i}^{T} \widetilde{\mathbf{K}}_{D, i} \boldsymbol{\phi}_{i}
$$


where the matrix $\widetilde{\mathbf{K}}_{D, i}$ is given by

$$
\widetilde{\mathbf{K}}_{D, i}=\mathbf{T}_{D}^{T} \operatorname{diag}\left\{\frac{\tau_{d, j}^{2} \omega_{i}^{2}}{1+\tau_{d, j}^{2} \omega_{i}^{2}} k_{d, j}\right\} \mathbf{T}_{D}
$$

It can be regarded as to the equivalent damping matrix for the $i$ th mode provided by added dampers. Combining Equations (2) and (8) yields the ith strain-energy ratio as

$$
\eta_{D, i}=\frac{\boldsymbol{\phi}_{i}^{T} \widetilde{\mathbf{K}}_{D k, i} \boldsymbol{\phi}_{i}}{\omega_{i}^{2}}
$$

Then, substitute Equation (2) into Equation (7), we can get

$$
\omega_{s, i}=\sqrt{1-\eta_{D, i}} \omega_{i}
$$

where $\omega_{s, i}$ is the $i$ th natural frequency of structure itself.

Finally, the equivalent natural frequency provided by dampers is given by

$$
\omega_{e q, i}=\omega_{i}-\sqrt{\left(1-\eta_{D, i}\right)} \omega_{i}
$$

It should be noted that Equations (6) and (12) require modal characteristics of passively controlled structures, including natural frequencies and complete mode shapes. However, in practice, civil engineering structures are often sparsely instrumented. As a result, their measured mode shapes are incomplete and correspond to a few monitored DOFs only. In this study, the mode shape expansion technique $[19,24,27]$ is adopted to obtain the complete mode shapes for the investigated building, as detailed in Section 4 .

\subsection{An Improved Berman-Nagy Method}

An improved direct updating method, called improved Berman-Nagy method, is presented here to correct the initial models of passively controlled structures themself. First, the traditional Berman-Nagy method [24] is utilized to demonstrate the incomplete modal matching issue, i.e., the predicted mode participation factors by updated models might not be consistent with those measured from real structures. Subsequently, a new procedure is proposed for mass matrix updating, in which the constraint of mode participation factors is considered.

\subsubsection{Problem Description}

Let $\mathbf{M}_{a}$ and $\mathbf{K}_{a}$ represent, respectively, the nominal mass and stiffness matrices of a structure with $n$ degrees of freedom. As discussed in the introduction part, its analytical modal characteristics frequently deviate from those measured from the real structure, with mass and stiffness matrices denoted as $\mathbf{M}$ and $\mathbf{K}$. In the traditional Berman-Nagy method, the updated mass matrix, denoted as $\mathbf{M}_{B}$, and stiffness matrix, denoted as $\mathbf{K}_{B}$, are

$$
\begin{gathered}
\mathbf{M}_{B}=\mathbf{M}_{a}+\mathbf{M}_{a} \boldsymbol{\Phi}\left(\boldsymbol{\Phi}^{\mathrm{T}} \mathbf{M}_{a} \boldsymbol{\Phi}\right)^{-1}\left(\mathbf{I}-\boldsymbol{\Phi}^{\mathrm{T}} \mathbf{M}_{a} \boldsymbol{\Phi}\right)\left(\boldsymbol{\Phi}^{\mathrm{T}} \mathbf{M}_{a} \boldsymbol{\Phi}\right)^{-1} \boldsymbol{\Phi}^{\mathrm{T}} \mathbf{M}_{a} \\
\mathbf{K}_{B}=\mathbf{K}_{a}+\mathbf{M}_{B} \boldsymbol{\Phi}\left(\boldsymbol{\Phi}^{\mathrm{T}} \mathbf{K}_{a} \boldsymbol{\Phi}+\boldsymbol{\Omega}^{2}\right) \boldsymbol{\Phi}^{\mathrm{T}} \mathbf{M}_{B}-\mathbf{K}_{a} \boldsymbol{\Phi} \boldsymbol{\Phi}^{\mathrm{T}} \mathbf{M}_{B}-\mathbf{M}_{B} \boldsymbol{\Phi} \boldsymbol{\Phi}^{\mathrm{T}} \mathbf{K}_{a}
\end{gathered}
$$

where $\Omega$ is the $m \times m$ diagonal matrix of measured natural frequencies, $m$ is the number of measured modes, $\boldsymbol{\Phi}$ is the $n \times m$ matrix of measured mode shapes, and $\mathbf{I}$ is the $m \times m$ identity matrix. Notably, 
such updated mass and stiffness matrices accurately replicate the measured natural frequencies and mode shapes, while they also generate predictions for mode participation factors as

$$
\mathbf{P}_{B}=\boldsymbol{\Phi}^{\mathrm{T}} \mathbf{M}_{B} \mathbf{I}_{n}=\left(\boldsymbol{\Phi}^{\mathrm{T}} \mathbf{M}_{a} \boldsymbol{\Phi}\right)^{-1} \boldsymbol{\Phi}^{\mathrm{T}} \mathbf{M}_{a} \mathbf{I}_{n}
$$

where $\mathbf{P}_{B}$ denotes the vector of predicted mode participation factors, $\mathbf{I}_{n}$ is the identity vector of order $n$. As discussed earlier, these byproduct predictions may differ from those measured from real structures. For applications in earthquake engineering, mode participation factors participate in determining mode response amplitudes, therefore, any error in their predictions will lead to flaws in updated structural models. To address this problem, a new procedure for mass matrix updating is developed as below.

\subsubsection{A New Procedure for Mass Matrix Updating}

This new procedure considers the constraints of symmetry, modal orthogonality, as well as mode participation factors in mass matrix updating. Its formulation process follows the guidance in reference [23]. Specifically, the cost function to be minimized is defined as

$$
\varepsilon=\left\|\mathbf{M}_{a}^{-1 / 2}\left(\mathbf{M}-\mathbf{M}_{a}\right) \mathbf{M}_{a}^{-1 / 2}\right\|
$$

subjected to constraints as follows:

$$
\begin{gathered}
\mathbf{M}=\mathbf{M}^{\mathrm{T}} \\
\boldsymbol{\Phi}^{\mathrm{T}} \mathbf{M} \boldsymbol{\Phi}=\mathbf{I} \\
\boldsymbol{\Phi}^{\mathrm{T}} \mathbf{M I}_{n}=\mathbf{P}
\end{gathered}
$$

where $\mathbf{P}$ is the vector of measured mode participation factors. Using the Lagrange multiplier method, the Lagrangian function may be formulated as

$$
J=\varepsilon+\sum_{i j} \Psi_{i j}\left(\mathbf{M}-\mathbf{M}^{\mathrm{T}}\right)_{i j}+\sum_{i j} \lambda_{i j}\left(\boldsymbol{\Phi}^{\mathrm{T}} \mathbf{M} \boldsymbol{\Phi}-\mathbf{I}\right)_{i j}+\sum_{i} \gamma_{i}\left(\boldsymbol{\Phi}^{\mathrm{T}} \mathbf{M} \mathbf{I}_{n}-\mathbf{P}\right)_{i}
$$

where $\Psi_{i j}, \lambda_{i j}$, and $\gamma_{i}$ are the undetermined multiplier coefficients, corresponding to the constraint equations above, and their matrix forms are denoted as $\Psi, \Lambda$, and $\Gamma$, respectively. Differentiating Equation (20) with respect to each element in $\mathbf{M}$ and setting these results equal to zero, it leads to

$$
\mathbf{M}=\mathbf{M}_{a}-\frac{1}{2} \mathbf{M}_{a}\left(\boldsymbol{\Phi} \Lambda \boldsymbol{\Phi}^{\mathrm{T}}+\boldsymbol{\Phi} \Gamma \mathbf{I}_{n}^{\mathrm{T}}+\mathbf{\Psi}-\mathbf{\Psi}^{\mathrm{T}}\right) \mathbf{M}_{a}
$$

Substituting Equation (21) into Equation (17) and arranging the result, the solution for $\Psi-\Psi^{\mathrm{T}}$ is

$$
\boldsymbol{\Psi}-\boldsymbol{\Psi}^{\mathrm{T}}=\frac{1}{2}\left(\boldsymbol{\Phi} \boldsymbol{\Lambda}^{\mathrm{T}} \boldsymbol{\Phi}^{\mathrm{T}}+\mathbf{I}_{n} \boldsymbol{\Gamma}^{\mathrm{T}} \boldsymbol{\Phi}^{\mathrm{T}}-\boldsymbol{\Phi} \boldsymbol{\Lambda} \boldsymbol{\Phi}^{\mathrm{T}}-\boldsymbol{\Phi} \boldsymbol{\Gamma} \mathrm{I}_{n}^{\mathrm{T}}\right)
$$

Inserting Equation (22) into Equation (21) to update mass matrix, it follows that

$$
\mathbf{M}=\mathbf{M}_{a}-\frac{1}{4} \mathbf{M}_{a} \boldsymbol{\Phi}\left(\boldsymbol{\Lambda}+\boldsymbol{\Lambda}^{\mathrm{T}}\right) \boldsymbol{\Phi}^{\mathrm{T}} \mathbf{M}_{a}-\frac{1}{4} \mathbf{M}_{a}\left(\boldsymbol{\Phi} \boldsymbol{\Gamma} \mathbf{I}_{n}^{\mathrm{T}}+\mathbf{I}_{n} \boldsymbol{\Gamma}^{\mathrm{T}} \boldsymbol{\Phi}^{\mathrm{T}}\right) \mathbf{M}_{a}
$$

Substitute Equation (23) into Equation (18), we can get

$$
\boldsymbol{\Lambda}+\boldsymbol{\Lambda}^{\mathrm{T}}=-4 \mathbf{m}_{a}^{-1}\left(\mathbf{I}-\mathbf{m}_{a}\right) \mathbf{m}_{a}^{-1}-\mathbf{\Gamma} \mathbf{P}_{a}^{\mathrm{T}} \mathbf{m}_{a}^{-1}-\mathbf{m}_{a}^{-1} \mathbf{P}_{a} \boldsymbol{\Gamma}^{\mathrm{T}}
$$


where $\mathbf{m}_{a}=\boldsymbol{\Phi}^{\mathrm{T}} \mathbf{M}_{a} \boldsymbol{\Phi}, \mathbf{P}_{\mathrm{a}}=\boldsymbol{\Phi}^{\mathrm{T}} \mathbf{M}_{\mathrm{a}} \mathbf{I}_{n}$. Eliminating the item $\boldsymbol{\Lambda}+\boldsymbol{\Lambda}^{\mathrm{T}}$ in Equation (23), mass matrix is rewritten as

$$
\mathbf{M}=\mathbf{M}_{B}+\frac{1}{4} \mathbf{M}_{a} \boldsymbol{\Phi} \Gamma\left(\mathbf{P}_{a}^{\mathrm{T}} \mathbf{m}_{a}^{-1} \boldsymbol{\Phi}^{\mathrm{T}}-\mathbf{I}_{n}^{\mathrm{T}}\right) \mathbf{M}_{a}+\frac{1}{4} \mathbf{M}_{a}\left(\boldsymbol{\Phi} \mathbf{m}_{a}^{-1} \mathbf{P}_{a}+\mathbf{I}_{n}\right) \boldsymbol{\Gamma}^{\mathrm{T}} \boldsymbol{\Phi}^{\mathrm{T}} \mathbf{M}_{a}
$$

Substitute Equation (25) into the last constraint, Equation (19), the solution for $\Gamma$ is

$$
\Gamma=\frac{4}{c} \mathbf{m}_{a}^{-1}\left(\mathbf{P}-\mathbf{P}_{B}\right)
$$

where $c=\left(\mathbf{P}_{a}^{\mathrm{T}} \mathbf{m}_{a}^{-1} \boldsymbol{\Phi}^{\mathrm{T}}-\mathbf{I}_{n}^{\mathrm{T}}\right) \mathbf{M}_{a} \mathbf{I}_{n}$ is a constant.

Finally, using Equation (26) to eliminate the matrix $\Gamma$ in Equation (25), the new, updated mass matrix is given by

$$
\mathbf{M}=\mathbf{M}_{B}+\Delta \mathbf{M}+\Delta \mathbf{M}^{\mathrm{T}}
$$

where the matrix $\Delta \mathbf{M}$ is

$$
\Delta \mathbf{M}=\frac{1}{c} \mathbf{M}_{a} \boldsymbol{\Phi} \mathbf{m}_{a}^{-1}\left(\mathbf{P}-\mathbf{P}_{B}\right)\left(\mathbf{P}_{a}^{\mathrm{T}} \mathbf{m}_{a}^{-1} \boldsymbol{\Phi}^{\mathrm{T}}-\mathbf{I}_{n}^{\mathrm{T}}\right) \mathbf{M}_{a}
$$

In addition, Using $\mathbf{M}$ to replace $\mathbf{M}_{B}$ in Equation (14), the new, updated stiffness matrix is

$$
\mathbf{K}=\mathbf{K}_{a}+\mathbf{M} \boldsymbol{\Phi}\left(\boldsymbol{\Phi}^{\mathrm{T}} \mathbf{K}_{a} \boldsymbol{\Phi}+\mathbf{\Omega}^{2}\right) \boldsymbol{\Phi}^{\mathrm{T}} \mathbf{M}-\mathbf{K}_{a} \boldsymbol{\Phi} \boldsymbol{\Phi}^{\mathrm{T}} \mathbf{M}-\mathbf{M} \boldsymbol{\Phi} \boldsymbol{\Phi}^{\mathrm{T}} \mathbf{K}_{a}
$$

Equations (27)-(29) form the improved Berman-Nagy method for mass and stiffness matrices updating. Notably, comparing Equations (13) and (27), $\Delta \mathbf{M}$ is the additional matrix rendering the new, updated mass matrix consistent with the measured mode participation factors. However, if $\mathbf{P}_{B}$ equal to $\mathbf{P}, \Delta \mathbf{M}$ will equal a zero matrix. In this instance, the improved Berman-Nagy method turns to the traditional Berman-Nagy method.

\section{Application to the Investigated Building}

The proposed framework is now implemented for the damping estimation of the investigated building using its earthquake measurements from the selected earthquake, as shown earlier in Figure 3. The finite element (FE) modeling of the building is first introduced to provide preliminary information for the following analysis. Subsequently, using the enhanced strain-energy method, the equivalent damping ratios and natural frequencies provided by oil dampers are estimated, and the modal parameters extracted from the earthquake measurements are modified accordingly. Finally, the improved Berman-Nagy method is utilized to update the initial FE model using the modified modal parameters. Moreover, the accuracy of these estimates is demonstrated through prediction validations.

Figure 4 shows a high-fidelity FE model of the building, initially established in the SAP2000 modeling environment based on the available architectural and structural drawings. The structure was assumed to be fixed on the ground floor without considering any soil-structure interaction effect. All steel columns, beams, and damper braces were modeled by using frame elements, while concrete floor slabs were modeled using flexible shell elements [32]. Following design guidance, the connections between structural components were assumed to be fully constrained. The oil dampers were modeled by linear Maxwell models with stiffness and damping parameters identified from their earthquake measurements, as detailed later. To reduce the computational complexity of this FE model, simplifications are adopted by invoking common assumptions used for building structures in earthquake engineering applications $[33,34]$. Note that the translational and torsional modes of this FE model are uncoupled due to the uniform distribution of mass and stiffness on floor plans, as illustrated in Section 2, which allows the analysis through planar models for each horizontal direction. This facilitates damping estimation and model updating of each direction separately, using the earthquake measurements for the corresponding motions of the building. Additionally, 
the building mass is assigned as lumped mass applied to beam-column joints at floor levels, while the column deformation in the vertical direction is not considered [34]. Therefore, rotational DoFs have zero mass, while vertical DoFs are neglected.

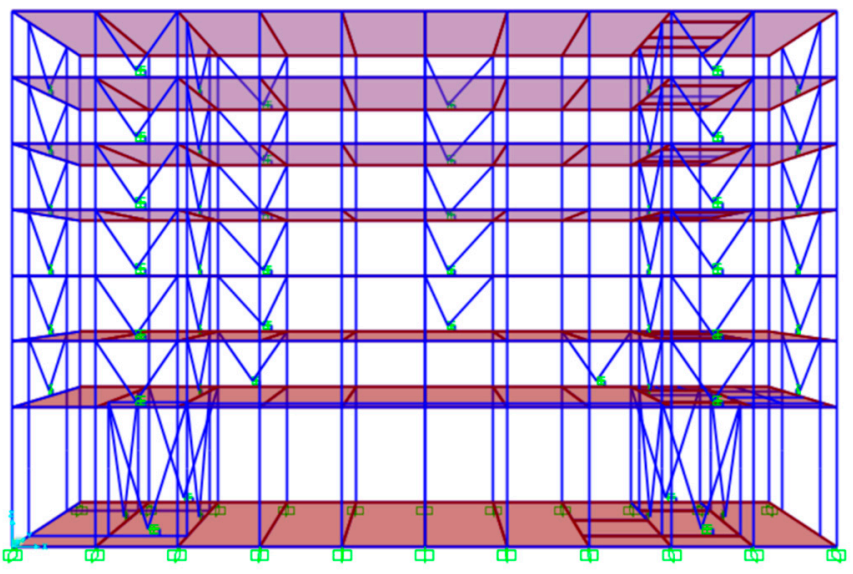

Figure 4. Finite element model of the building as developed in SAP2000 environment.

The resulting planar FE models have 7 translational DoFs (with mass) and 279 (zero-mass) rotational DoFs for the beam-column joints. Static condensation [33] is further performed to remove the zero-mass rotational DoFs. The resulting nominal mass matrix is $\mathbf{M}_{a}=$ diag $\{446348337334328$ $324311\} /$ ton for the two horizontal directions, where diag stands for a diagonal matrix with elements included in the bracket $\{\cdot\}$, while the initial stiffness matrices(unit: $\mathrm{MN} / \mathrm{mm}$ ) are

$$
\begin{aligned}
& \mathbf{K}_{a}=\left[\begin{array}{lrlrlrl}
1.076 & -1.081 & 0.290 & -0.047 & 0.007 & -0.001 & 0.000 \\
-1.081 & 1.895 & -1.163 & 0.313 & -0.052 & 0.008 & -0.001 \\
0.290 & -1.163 & 1.732 & -1.101 & 0.296 & -0.047 & 0.006 \\
-0.047 & 0.313 & -1.101 & 1.652 & -1.055 & 0.271 & -0.035 \\
0.007 & -0.052 & 0.296 & -1.055 & 1.565 & -0.963 & 0.201 \\
-0.001 & 0.008 & -0.047 & 0.271 & -0.963 & 1.357 & -0.625 \\
0.000 & -0.001 & 0.006 & -0.035 & 0.201 & -0.625 & 0.453
\end{array}\right] \text { for longitudinal direction }(30) \\
& \mathbf{K}_{a}=\left[\begin{array}{lrlrlrl}
1.084 & -1.077 & 0.278 & -0.043 & 0.006 & -0.001 & 0.000 \\
-1.077 & 1.091 & -1.160 & 0.293 & -0.047 & 0.007 & -0.000 \\
0.278 & -1.160 & 1.756 & -1.101 & 0.278 & -0.044 & 0.005 \\
-0.043 & 0.293 & -1.101 & 1.679 & -1.058 & 0.262 & -0.033 \\
0.006 & -0.047 & 0.278 & -1.058 & 1.592 & -0.965 & 0.193 \\
-0.001 & 0.007 & -0.044 & 0.262 & -0.965 & 1.372 & -0.631 \\
0.000 & -0.001 & 0.005 & -0.033 & 0.193 & -0.631 & 0.466
\end{array}\right] \text { for transversal direction (31) }
\end{aligned}
$$

The corresponding condensed models are referred to as the initial models, which are utilized in the following analysis. The equation of motion of the building in each of the two translational directions may be expressed by

$$
\mathbf{M} \ddot{\mathbf{x}}+\mathbf{C} \dot{\mathbf{x}}+\mathbf{K} \mathbf{x}+\mathbf{T}_{D}^{\mathrm{T}} \mathbf{f}_{D}\left(\mathbf{x}, \dot{\mathbf{x}}, \mathbf{c}_{d}, \mathbf{k}_{d}\right)=\mathbf{M I} \ddot{x}_{g}
$$

where $\mathbf{M}, \mathbf{K}$, and $\mathbf{C}$ are, respectively, the real mass, stiffness, and inherent damping matrices, $\mathbf{x}$ represents the vector of floor displacement relative to the structure base, $\ddot{x}_{g}$ is the acceleration input, corresponding to the acceleration of the ground floor of the building, I is the vector of earthquake influence coefficients, $\mathbf{f}_{D}(\cdot)$ denotes the vector of damper forces, $\mathbf{T}_{D}$ is the transformation matrix, relating movement across the ends of each damper to $\mathbf{x}$, and $\mathbf{c}_{d}$, and $\mathbf{k}_{d}$ are the vectors of damping coefficients and stiffness 
parameters of linear Maxwell models, respectively, composed of the respective characteristics of the oil dampers for each floor. The damper force, for example, for the $i$ th floor damper is expressed as

$$
\frac{\dot{f}_{d, i}}{k_{d, i}}+\frac{f_{d, i}}{c_{d, i}}=\dot{x}_{d, i}
$$

where $f_{d, i}$ and $x_{d, i}$ are, respectively, the damper force and the damper deformation between its two ends. For the investigated building, the supporting brace stiffness is estimated based on their design information and is much larger than the identified damper stiffness. Since these two components are connected in series, the damper deformation is taken as the corresponding inter-story drift. Besides, note that a representation of the form of Equation (32) holds separately for each of the horizontal directions, though for notational simplicity, this distinction is not explicitly noted herein.

\subsection{Damping Estimation of the Building}

Implementations of the enhanced strain-energy method require the building modal data and the oil damper parameters. These characteristics are identified by adopting a standard time-domain identification technique $[7,35]$ and using the selected earthquake measurements. The identification is separately performed in each horizontal direction, which facilitates a better consideration of the impact of the excitation/response amplitude for oil dampers. Precisely, for oil damper identification, the input-output earthquake data corresponds to the input displacement and output force data, with the governing equation given by Equation (33), while, for modal identification, the data is the acceleration of the ground floor (input) and two monitored floors (output), with modal-superposition models as governing equation [35].

Table 1 lists the identification results for the four monitored oil dampers, in which I and II represent the damper types. Recall that Type I oil dampers were installed on the ground floor, while Type II oil dampers were placed on other upper floors. It is evident from the results that a discrepancy exists between the identified parameters of same-type dampers, verifying their anticipated amplitude-dependent characteristics. Table 2 shows the identified (translational) modal parameters of the first two modes of the building. The remaining higher modes are of small importance, and identification of their properties suffers to a much larger degree by measurement noise [35]. Notably, the identified mode shapes and mode participation factors in Table 2 are determined by using the so-called mode scale factor, which is commonly adopted to (mass) normalize measured mode shapes. Furthermore, to obtain complete mode shapes, an expansion technique suggested in Reference [27] is adopted, though note that other alternatives exist for resolving this issue [19]. The implementations of this expansion approach are omitted here since it is a common practice. For clarity of explanation, the expanded mode shape is denoted hereinafter as $\phi_{i}$, for the $i$ th mode of the building.

Table 3 shows the estimated equivalent damping ratios $\left(\xi_{\text {eq }, i}\right)$ and natural frequencies $\left(\omega_{\text {eq }, i}\right)$ by the enhanced strain-energy method. Results of the inherent damping ratios $\left(\xi_{s, i}\right)$ and natural frequencies $\left(\omega_{s, i}\right)$ for the building itself are also reported in this table. They are obtained by subtracting those equivalent estimates from the corresponding identified modal parameters in Table 2. It can be seen from Table 3 that the estimated equivalent damping ratios have a sharp decrease when mode order increases and the second equivalent damping ratios are negligible. It indicates that the energy dissipated by oil dampers concentrates in the seismic motion of the fundamental modes. On the other side, for equivalent natural frequencies, an increasing trend is observed. Overall, they are relatively small compared with the identified natural frequencies of the building. However, it does not indicate that the estimated equivalent natural frequencies are unimportant. Instead, they can significantly affect the prediction accuracy of updated models, as will be shown in the next section. 
Table 1. The identified model parameters for monitored oil dampers.

\begin{tabular}{ccccc}
\hline \multirow{2}{*}{ Parameters } & \multicolumn{2}{c}{$\begin{array}{c}\text { Longitudinal Direction } \\
\text { Type I }\end{array}$} & Type II & \multicolumn{2}{c}{ Transversal Direction } \\
& Type I & Type II \\
\hline$c_{d}\left(\mathrm{kN} / \mathrm{mm} \cdot \mathrm{s}^{-1}\right)$ & 0.696 & 0.685 & 0.876 & 0.516 \\
$k_{d}(\mathrm{kN} / \mathrm{mm})$ & 4.258 & 2.562 & 3.395 & 3.295 \\
\hline
\end{tabular}

Table 2. The identified modal parameters for the building.

\begin{tabular}{ccccc}
\hline \multirow{2}{*}{ Parameters } & \multicolumn{2}{c}{ Longitudinal Direction } & \multicolumn{2}{c}{ Transversal Direction } \\
& 1st Mode & 2nd Mode & 1st Mode & 2nd Mode \\
\hline$\omega_{i}(\mathrm{rad} / \mathrm{s})$ & 5.608 & 18.132 & 5.062 & 16.552 \\
$\xi_{i}$ & 0.031 & 0.061 & 0.036 & 0.053 \\
$\phi_{i, 2}$ & 0.014 & -0.026 & 0.013 & -0.026 \\
]$\phi_{i, 6}$ & 0.025 & 0.016 & 0.026 & 0.016 \\
$p_{i}$ & 47.421 & -15.392 & 48.952 & -15.324 \\
\hline
\end{tabular}

Table 3. The estimated equivalent natural frequencies and equivalent damping ratios provided by oil dampers. The corresponding parameters of the building itself also shown.

\begin{tabular}{ccccc}
\hline \multirow{2}{*}{ Parameters } & \multicolumn{2}{c}{ Longitudinal Direction } & \multicolumn{2}{c}{ Transversal Direction } \\
& 1st Mode & 2nd Mode & 1st Mode & 2nd Mode \\
\hline$\omega_{s, i}(\mathrm{rad} / \mathrm{s})$ & 5.495 & 17.721 & 4.951 & 16.108 \\
$\omega_{e q, i}(\mathrm{rad} / \mathrm{s})$ & 0.113 & 0.411 & 0.111 & 0.444 \\
$\xi_{s, i}$ & 0.012 & 0.055 & 0.017 & 0.045 \\
$\xi_{\text {eq }, i}$ & 0.019 & 0.006 & 0.019 & 0.008 \\
\hline
\end{tabular}

Besides, the enhanced strain-energy method also allows quantifying the contribution from a specific damper to the equivalent damping ratios and natural frequencies, as evident from Equations (3) and (8). Figure 5 plots the damping and stiffness contribution in percentages from the oil dampers of different floors along with the two horizontal directions. The dampers on the first floor dominate the contribution, up to $70 \%$ for damping and up to $50 \%$ for stiffness, which is attributed to its larger story drift. Because the damping and stiffness contribution from oil dampers is proportional to the square values of $\phi_{i, d j}$, as indicated in Equations (3) and (8). Higher floor dampers contribute more to the damping of the second mode, but the total damping contribution in that mode is negligible, as shown in Table 3.

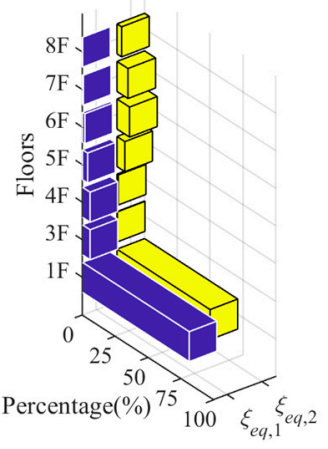

(a)

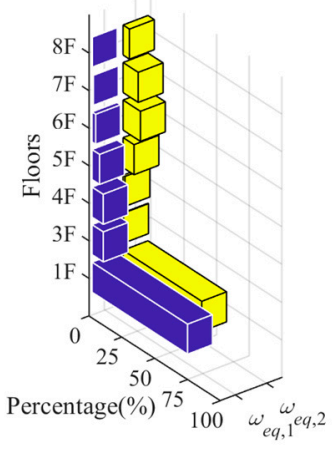

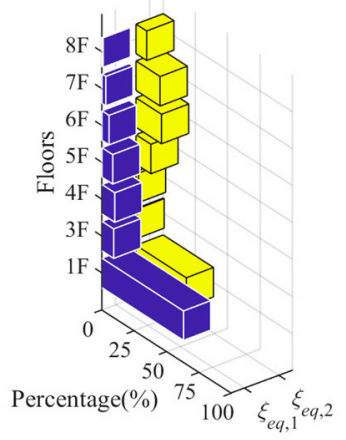

(b)

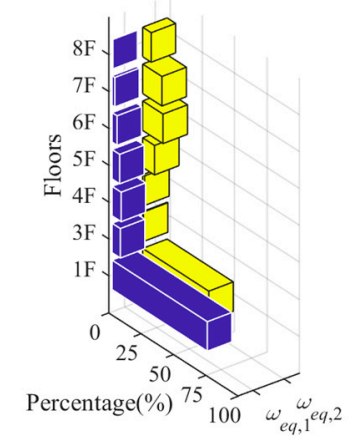

(b)

Figure 5. Percentages of the equivalent damping ratios and natural frequencies from oil dampers of same floors: (a) Longitudinal direction; (b) Transversal direction.

It is important to stress once more that the enhanced strain-energy method is built based on the assumptions of harmonic motion and mode-proportional deformation of structure and added dampers, 
which violate their real situations during earthquakes. Therefore, it is necessary to verify the method accuracy, which is achieved through model updating and prediction validations.

\subsection{Model Updating of the Building}

The model updating of the building itself is separately performed for its two horizontal directions. Specifically, for each updating case, the natural frequencies $\left(\omega_{s, i}\right)$, expanded mode shapes $\left(\phi_{i}\right)$, and mode participation factors $\left(p_{i}\right)$ of the first two modes are regrouped and utilized to correct mass and stiffness matrices by the improved Berman-Nagy method, i.e., Equation (27) for mass matrix updating and Equation (29) for stiffness matrix updating. Besides, for inherent structural damping, the damping matrix is modeled through a common modal damping assumption and is adjusted to match the estimated damping ratios $\left(\xi_{s, i}\right)$, as listed in Table 3. Damping ratios for the remaining higher modes are generally large and are constrained to a predefined value (taken in the case study later as $10 \%$ ). Note that using a large damping ratio for these modes, though not critical, can efficiently suppress higher-frequency components in the building response predictions.

First, mass and stiffness matrices are updated to demonstrate the effectiveness of the improved Berman-Nagy method. Two updating cases of using different modal data are considered to illustrate the impact of additional matrix $\Delta \mathbf{M}$ on updated mass matrix. Specifically, the regrouped modal parameters of the first mode and the first two modes are separately utilized to update the nominal mass matrix $\mathbf{M}_{a}$. Figure 6 presents the new, updated mass matrix $\mathbf{M}$ by Equation (27). For comparisons, the updated mass matrix $\mathbf{M}_{B}$ by the traditional Berman-Nagy method is also reported. The results in this figure correspond to the transversal direction FE model.
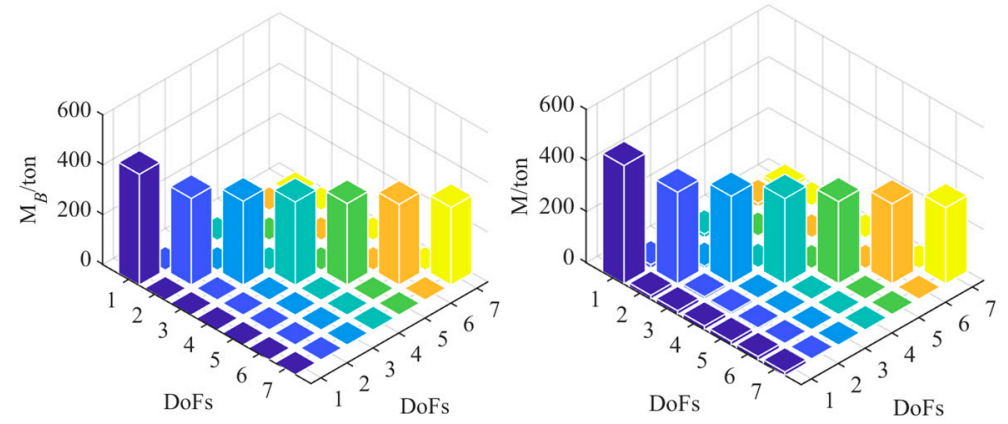

(a)
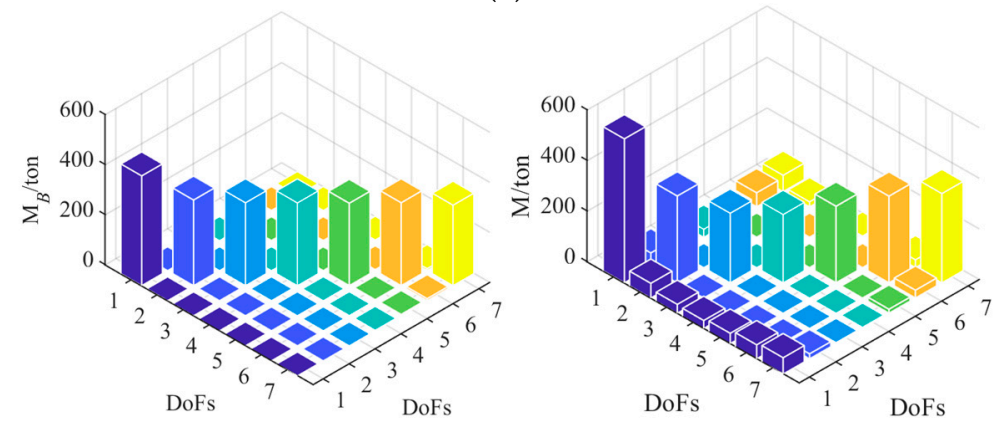

(b)

Figure 6. Updated mass matrices by Equations (13) and (27): (a) updating results by using the first mode; (b) updating results by using the first two modes.

It is evident from the results in Figure 6 that $\Delta \mathbf{M}$, the difference between $\mathbf{M}$ and $\mathbf{M}_{B}$, has an increasing impact on the updated mass matrix when more modal data is utilized. In the first updating case, the predicted mode participation factor by $\mathbf{M}$ exactly matches the measured one (48.952), while the prediction by $\mathbf{M}_{B}$ is 46.884 . This prediction error is large but causes small changes in the two updated mass matrices, as shown in Figure 6a. When the regrouped modal data of the first two modes is 
utilized, $\mathbf{M}$ and $\mathbf{M}_{B}$ differ significantly in both diagonal and off-diagonal elements. The predicted mode participation factors are listed in Table 4, where the improved method results are denoted as $p_{I, i}$. Again, $\mathbf{M}$ reproduces exactly the measured mode participation factors, while $\mathbf{M}_{B}$ fails in the first two modes cases. Figure 7 shows the new, updated stiffness matrices by the improved method and using the regrouped modal data of the first two modes. Unlike updated mass matrices, changes in updated stiffness matrices are small. Notably, the updated mass and stiffness matrices by the improved method exactly possess the modal data, including natural frequencies, mode shapes, and mode participation factors.

Table 4. Comparisons of the predicted mode participation factors by updated mass matrices.

\begin{tabular}{ccccc}
\hline \multirow{2}{*}{ Parameters } & \multicolumn{2}{c}{ Longitudinal Direction } & \multicolumn{2}{c}{ Transversal Direction } \\
& 1st Mode & 2nd Mode & 1st Mode & 2nd Mode \\
\hline$p_{B, i}$ & 45.8910 & -12.0991 & 45.9156 & -14.0551 \\
$p_{I, i}$ & 47.4219 & -15.3921 & 48.9527 & -15.3241 \\
\hline
\end{tabular}

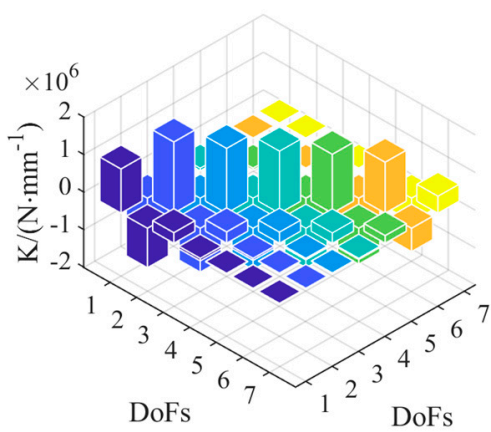

(a)

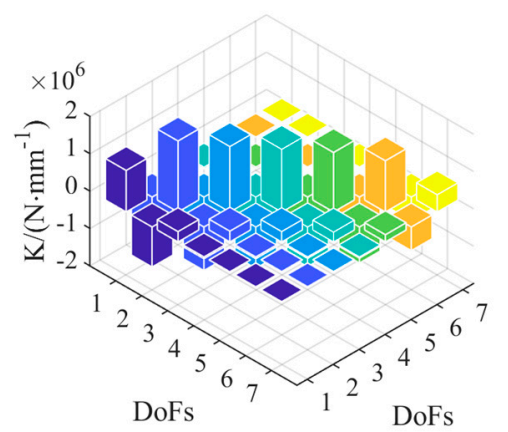

(b)

Figure 7. Updated stiffness matrices for the building itself: (a) Longitudinal direction; (b) Transversal direction.

Then, the precision of the estimated inherent damping ratios $\left(\xi_{s, i}\right)$ and natural frequencies $\left(\omega_{s, i}\right)$ is considered. The updated models with new, updated mass and stiffness matrices are utilized for the building response prediction, according to Equation (34). The linear Maxwell models with identified parameters shown in Table 1 are assigned to the same-type oil dampers, i.e., the parameter configures of Type I for the ground-floor oil dampers and those of Type II for the other floors oil dampers. Two updated models are utilized for this response prediction: In the first updated model, the damper stiffness is not considered, the modal data utilized for correcting mass and stiffness matrices is $\left\{\omega_{i}, \phi_{i}\right.$, $\left.p_{i}, \xi_{s, i}, i=1,2\right\}$, while, in the second updated model, the data is $\left\{\omega_{s, i}, \boldsymbol{\phi}_{i}, p_{i}, \xi_{s, i}, i=1,2\right\}$. To distinguish them, they are labeled by updated model I and II, respectively.

Figures 8 and 9 present the measured and predicted floor acceleration and damper force, respectively. The results in these figures correspond to the longitudinal direction updated models. For clarity of illustration, the response predictions during the strong shaking phase (30s 60 s) are shown, and the results of the two updated models are plotted separately. The normalized root mean squared error (NRMSE) between the measured and predicted response, defined as

$$
e=\sqrt{\operatorname{mean}\left[(y-\widetilde{y})^{2}\right]} / \sqrt{\operatorname{mean}\left[\widetilde{y}^{2}\right]}
$$

is utilized to quantify the goodness of fit between them, in which $\widetilde{y}$ and $\mathrm{y}$ are the measured and predicted response, respectively. The results for the two updated models are listed in Table 5. 


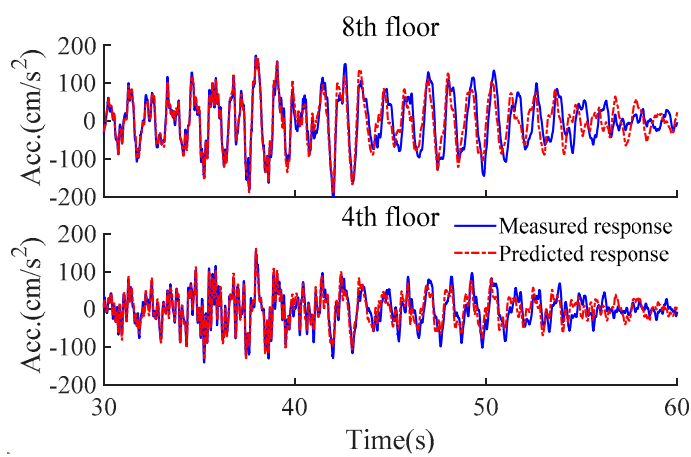

(a)

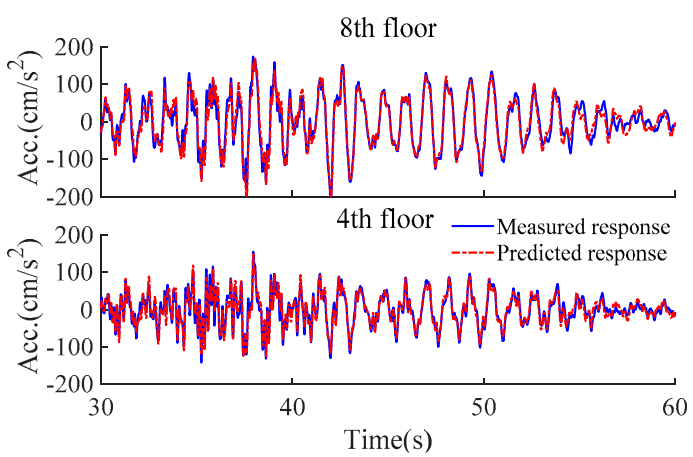

(b)

Figure 8. Comparison of the predicted and measured floor acceleration: (a) updated model I; (b) updated model II.

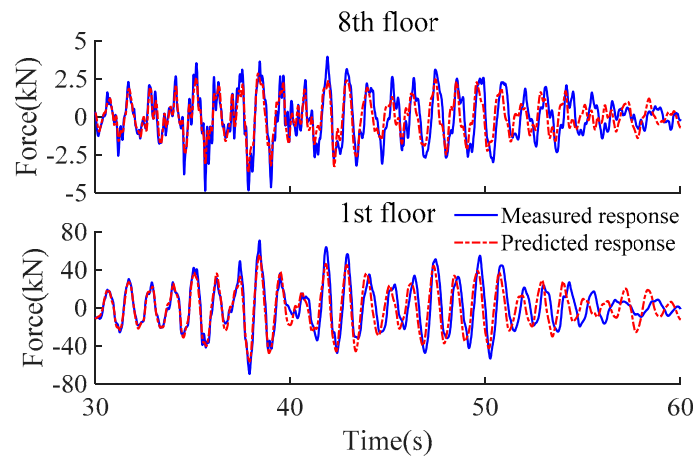

(a)

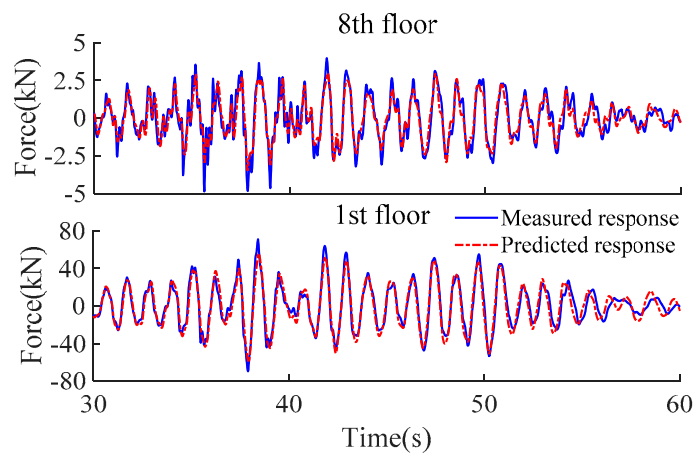

(b)

Figure 9. Comparison of the predicted and measured damper force: (a) updated model I; (b) updated model II.

Table 5. Normalized root mean squared errors (NRMSEs) of the response predictions of updated models.

\begin{tabular}{cccccccccc}
\hline \multirow{2}{*}{$\begin{array}{c}\text { Updated } \\
\text { Models }\end{array}$} & \multicolumn{3}{c}{ Acceleration } & \multicolumn{2}{c}{ Damper Force } & \multicolumn{4}{c}{ Transversal Direction } \\
& $\mathbf{4 F}$ & $\mathbf{8 F}$ & $\mathbf{1 F}$ & $\mathbf{8 F}$ & $\mathbf{4 F}$ & $\mathbf{8 F}$ & $\mathbf{1 F}$ & $\mathbf{8 F}$ \\
\hline Model I & 0.468 & 0.466 & 0.597 & 0.709 & 0.461 & 0.308 & 0.536 & 0.641 \\
Model II & 0.278 & 0.225 & 0.267 & 0.392 & 0.236 & 0.118 & 0.268 & 0.444 \\
\hline
\end{tabular}

It can be seen from Figures 8 and 9 that the predicted responses by updated model II match very well the measured data with NRMSE values close to 0.2 , which indicates a high precision in response prediction. On the other side, the updated model I has non-negligible phase-lags errors in all response predictions with larger NRMSE values up to 0.7. A similar trend of calculated NRMSEs is also observed for the transversal direction updated models, as shown in Table 5. It verifies that, at least in this instance, the damper stiffness or the estimated equivalent natural frequencies, though small, can not be omitted in model updating for the building itself. Moreover, the high precision predictions of updated model II also show that the estimated equivalent damping ratios and natural frequencies are accurate, which addresses the concern at the end of the last section.

\section{Conclusions}

This paper examined the damping estimation of an eight-story steel building structure equipped with oil dampers, located at Tohoku Institute of Technology. It was carried out within a proposed framework that consists of an enhanced strain-energy method and an improved Berman-Nagy method for model updating. The former method was first employed to quantify the additional damping and stiffness provided by oil dampers in the form of equivalent damping ratios and natural frequencies. 
Subsequently, the improved Berman-Nagy was implemented to update an initial model of the building by using its estimated modal parameters, whose accuracy were further verified through prediction validations. The case study demonstrated the effectiveness of the proposed framework. The main conclusions and findings obtained from this study are the following: (1) The updated models show a high precision in prediction for both floor acceleration and damper force, showing the estimated natural frequencies and damping ratios are accurate. Besides, the damper stiffness, though not large, can significantly worsen the model prediction precision and, therefore, should be taken into consideration in model updating. (2) The updated model by the improved Berman-Nagy method reproduces exactly the measured modal data, including mode participation factors. However, the non-diagonal elements of updated mass matrix are not negligible and become large when more modal data utilized. (3) The supplemental damping ratios for the first modes are about 0.02 , while the damping contributions for second modes are negligible, indicating that most energy dissipated by oil dampers concentrates in the seismic motion of fundamental modes. (4) The proposed framework and methods are computationally efficient and have good potential in the post-earthquake health and safety assessment for passively controlled building structures.

Author Contributions: Conceptualization, P.Y. and S.X.; methodology, P.Y.; software, L.X. and M.C.; validation, P.Y.; formal analysis, P.Y.; writing—original draft preparation, P.Y.; writing—review and editing, P.Y. and L.X. All authors have read and agreed to the published version of the manuscript.

Funding: This research was funded by the National Natural Science Foundation of China (Grant No. 51778490 and 51978525), the Natural Science Foundation of Shanghai (Grant No. 20ZR1461800), the Japan Society for Promotion of Science(Grant No. 18K04438 and 19F19777), and the Tohoku Institute of Technology research Grant.

Conflicts of Interest: The authors declare no conflict of interest.

\section{References}

1. Symans, M.D.A.; Charney, F.A.; Whittaker, A.S.; Constantinou, M.C.; Kircher, C.A.; Johnson, M.W.; McNamara, R.J. Energy dissipation systems for seismic applications: Current practice and recent developments. J. Struct. Eng. 2008, 134, 3-21. [CrossRef]

2. Soong, T.T.; Spencer, B.F., Jr. Supplemental energy dissipation: State-of-the-art and state-of-the-practice. Eng. Struct. 2002, 24, 243-259. [CrossRef]

3. Ikago, K.; Saito, K.; Inoue, N. Seismic control of single-degree-of-freedom structure using tuned viscous mass damper. Earthq. Eng. Struct. Dyn. 2012, 41, 453-474. [CrossRef]

4. Hwang, J.S.; Tsai, C.H.; Wang, S.J.; Huang, Y.N. Experimental study of RC building structures with supplemental viscous dampers and lightly reinforced walls. Eng. Struct. 2006, 28, 1816-1824. [CrossRef]

5. Kasai, K.; Ito, H.; Ooki, Y.; Hikino, T.; Kajiwara, K.; Motoyui, S. Full scale shake table tests of 5-story steel building with various dampers. In Proceedings of the 7th International Conference on Urban Earthquake Engineering \& 5th International Conference on Earthquake Engineering, Tokyo, Japan, 3-5 March 2010.

6. Santarsiero, G.; Di Sarno, L.; Giovinazzi, S.; Masi, A.; Cosenza, E.; Biondi, S. Performance of the healthcare facilities during the 2016-2017 Central Italy seismic sequence. Bull. Earthq. Eng. 2019, 17, 5701-5727. [CrossRef]

7. Greco, R.; Marano, G.C. Identification of parameters of Maxwell and Kelvin-Voigt generalized models for fluid viscous dampers. J. Vib. Control. 2015, 21, 260-274. [CrossRef]

8. Hazaveh, N.K.; Rodgers, G.W.; Chase, J.G.; Pampanin, S. Experimental test and validation of a direction-and displacement-dependent viscous damper. J. Eng. Mech. 2017, 143, 04017132. [CrossRef]

9. Bixio, A.R.; Braga, F.; Dolce, M.; Nicoletti, M.; Nigro, D.; Ponzo, F.C. Repeatable dynamic release tests on a base-isolated building. J. Earthq. Eng. 2001, 5, 369-393. [CrossRef]

10. Ierimonti, L.; Venanzi, I.; Cavalagli, N.; Comodini, F.; Ubertini, F. An innovative continuous Bayesian model updating method for base-isolated RC buildings using vibration monitoring data. Mech. Syst. Signal Process. 2020, 139, 106600. [CrossRef]

11. Inman, D.J.; Farrar, C.R.; Junior, V.L.; Junior, V.S. Damage Prognosis: For Aerospace, Civil and Mechanical Systems; John Wiley \& Sons: Chichester, UK, 2005; pp. 131-198. 
12. Bartoli, G.; Betti, M.; Marra, A.M.; Monchetti, S. A Bayesian model updating framework for robust seismic fragility analysis of non-isolated historic masonry towers. Philos. Trans. R. Soc. A 2019, 377, 20190024. [CrossRef]

13. Zhang, R.H.; Soong, T.T.; Mahmoodi, P. Seismic response of steel frame structures with added viscoelastic dampers. Earthq. Eng. Struct. Dyn. 1989, 18, 389-396. [CrossRef]

14. Chang, K.C.; Lai, M.L.; Soong, T.T.; Hao, D.S.; Yeh, Y.C. Seismic Behavior and Design Guidelines for Steel Frame Structures with Added Viscoelastic Dampers; National Center for Earthquake Engineering Research: Buffalo, NY, USA, 1993; pp. 21-86.

15. Ji, X.; Hikino, T.; Kasai, K.; Nakashima, M. Damping identification of a full-scale passively controlled five-story steel building structure. Earthq. Eng. Struct. Dyn. 2013, 42, 277-295. [CrossRef]

16. Fournier, J.A.; Cheng, S. Impact of damper stiffness and damper support stiffness on the efficiency of a linear viscous damper in controlling stay cable vibrations. J. Bridge Eng. 2014, 19, 04013022. [CrossRef]

17. Chen, Y.T.; Chai, Y.H. Effects of brace stiffness on performance of structures with supplemental Maxwell model-based brace-damper systems. Earthq. Eng. Struct. Dyn. 2011, 40, 75-92. [CrossRef]

18. Aghagholizadeh, M.; Catbas, F.N. A review of model updating methods for civil infrastructure systems. In Computational Techniques for Civil and Structural Engineering; Kruis, J., Tsompanakis, Y., Topping, B.H.V., Eds.; Saxe-Coburg Publications: Stirlingshire, Scotland, 2015; pp. 83-99.

19. Friswell, M.; Mottershead, J.E. Finite Element Model Updating in Structural Dynamics; Springer Science \& Business Media: Berlin, Germany, 1996; pp. 56-155.

20. Marwala, T. Finite Element Model Updating Using Computational Intelligence Techniques: Applications to Structural Dynamics; Springer Science \& Business Media: Berlin, Germany, 2010; pp. 1-231.

21. Abdullah, N.A.; Sani, M.S.; Rahman, M.M.; Zaman, I. A review on model updating in structural dynamics. In IOP Conference Series: Materials Science and Engineering; IOP Publishing: Kuantan, Malaysia, 2015.

22. Baruch, M.; Bar-Itzhack, I.Y. Optimal weighted orttiogonalization of measured modes. AIAA J. 1978, 16, 346-351. [CrossRef]

23. Berman, A. Mass matrix correction using an incomplete set of measured modes. AIAA J. 1979, 17, 1147-1148. [CrossRef]

24. Berman, A.; Nagy, E.J. Improvement of a large analytical model using test data. AIAA J. 1983, 21, 1168-1173. [CrossRef]

25. Caesar, B.; Peter, J. Direct update of dynamic mathematical models from modal test data. AIAA J. 1987, 25, 1494-1499. [CrossRef]

26. Wei, F.S. Analytical dynamic model improvement using vibration test data. AIAA J. 1990, $28,175-177$. [CrossRef]

27. Yuen, K.V. Updating large models for mechanical systems using incomplete modal measurement. Mech. Syst. Signal Process. 2012, 28, 297-308. [CrossRef]

28. Lee, E.T.; Eun, H.C. Update of corrected stiffness and mass matrices based on measured dynamic modal data. Appl. Math. Model. 2009, 33, 2274-2281. [CrossRef]

29. Yang, Y.B.; Chen, Y.J. A new direct method for updating structural models based on measured modal data. Eng. Struct. 2009, 31, 32-42. [CrossRef]

30. Kawamata, S.; Funaki, N.; Itoh, Y. Passive control of building frames by means of liquid dampers sealed by viscoelastic material. In Proceedings of the 12th World Conference on Earthquake Engineering, Auckland, New Zealand, 1-4 February 2000.

31. Lee, S.H.; Min, K.W.; Hwang, J.S.; Kim, J. Evaluation of equivalent damping ratio of a structure with added dampers. Eng. Struct. 2004, 26, 335-346. [CrossRef]

32. De Angelis, A.; Pecce, M.R. The structural identification of the infill walls contribution in the dynamic response of framed buildings. Struct. Control Health Monit. 2019, 26, e2405. [CrossRef]

33. Chopra, A.K. Dynamics of Structures: Theory and Applications to Earthquake Engineering, 6th ed.; Prentice Hall: Englewood Cliffs, NJ, USA, 2007; pp. 255-455.

34. Behmanesh, I.; Yousefianmoghadam, S.; Nozari, A.; Moaveni, B.; Stavridis, A. Uncertainty quantification and propagation in dynamic models using ambient vibration measurements, application to a 10-story building. Mech. Syst. Signal Process. 2018, 107, 502-514. [CrossRef] 
35. Cruz, C.; Miranda, E. Evaluation of the Rayleigh damping model for buildings. Eng. Struct. 2017, 138, 324-336. [CrossRef]

Publisher's Note: MDPI stays neutral with regard to jurisdictional claims in published maps and institutional affiliations.

(C) 2020 by the authors. Licensee MDPI, Basel, Switzerland. This article is an open access article distributed under the terms and conditions of the Creative Commons Attribution (CC BY) license (http://creativecommons.org/licenses/by/4.0/). 